\title{
Lung Region Segmentation using Artificial Neural Network Hopfield Model for Cancer Diagnosis in Thorax CT Images
}

\author{
Rachid Sammouda, Hassan Ben Mathkour \\ Computer Science Department, College of Computer and Information Sciences, King Saud University, Riyadh, Saudi Arabia
}

Email address:

rsammouda@ksu.edy.sa (R. Sammouda)

To cite this article:

Rachid Sammouda, Hassan Ben Mathkour. Lung Region Segmentation using Artificial Neural Network Hopfield Model for Cancer Diagnosis in Thorax CT Images. Automation, Control and Intelligent Systems. Vol. 3, No. 2, 2015, pp. 19-25.

doi: 10.11648/j.acis.20150302.12

\begin{abstract}
Lung Cancer was found to be one of the leading causes of death of human persons throughout the world. It spreads rapidly after it forms. The survival rate of patient is very low as the disease is identified in a very late stage. In this paper, we represent a fully automated and three-dimensional segmentation method for the early identification of cancerous pixels in thorax Computed Tomography database. The segmentation process is meant to be considered as the bottleneck in the Computer Aided Diagnosis system for lung cancer detection based on the Computed Tomography pixels' values. We have formulated the segmentation problem as the optimization of a certain energy function. A special Classifier was designed using Hopfield Artificial Neural Network in order to classify or segment the set of pixels in the CT images of the Thorax into a set of user decided number of regions. A step function was designed, implemented and tested to ensure a high convergence speed of the classifier to local optimum that is close to the global optima. The lung contour was adequately located in $95 \%$ of the CT scans using a pre-segmentation process based on bit-planes' features of the CT scans. The segmentation process was initially developed and tested on a large dataset of subjects, with normal and abnormal lung tissues at different stages, each of $150 \mathrm{CT}$ scans giving very satisfactory results.
\end{abstract}

Keywords: Chest CT Images, Lung Cancer, Segmentation

\section{Introduction}

Lung cancer is considered to be as the principal cause of cancer decease all over the world and it is hard to be identified at primary phases since its symptoms appear only at progressive phases [1]. In Saudi Arabia, Lungs cancer is ranked fourth in the number of deaths related with cancer [2]. In most cases tumor cells do not show any symptoms at early stages and radiologists will play a critical role in the effective and responsible implementation of Computer Tomography (CT) based lung cancer screening. Such diagnostic method is the only technique recognized to date that permits preclinical finding of lung cancer such that suitable interference considerably decreases lung cancer death rate.

Segmentation of pulmonary regions using Chest CT mages is a forerunner to any pulmonary image examination application or Computer Aide Diagnosis (CAD) system. Here we present a fully automatic process for segmenting the lung in three-dimensional (3-D) CT images. The process has two leading phases. First, the lung region is extracted from the
CT images by applying different image processing techniques such as Bit-Plane Slicing, Erosion, Median Filter, Dilation, Outlining, Lung Border Extraction and Flood-Fill algorithms for extraction of lung region. Then for segmentation Hopfield Artificial Neural Network Classifier (HANNC) is used in order to classify the lung pixels, based on their $\mathrm{CT}$ values in the raw $\mathrm{CT}$ images of the Thorax, into a set of user decided number of regions.

The method has been tested by processing 3-D CT data sets from normal and abnormal subjects.

\section{Methods}

The input data of the segmentation process is a 3-D CT data set organized as a stack of 2-D transverse slices of $2 \mathrm{~mm}$ thin. It represents the thorax area from the trachea to below the diaphragm as shown in Figure1.

In the $\mathrm{CT}$ chest data, air will appear with a mean intensity 
of approximately -1000 Hounsfield units (HU), most lung tissues range of $-910 \mathrm{HU}$ to $-500 \mathrm{HU}$, while the chest wall, blood, and bone will be much dense (well above $-500 \mathrm{HU}$ )

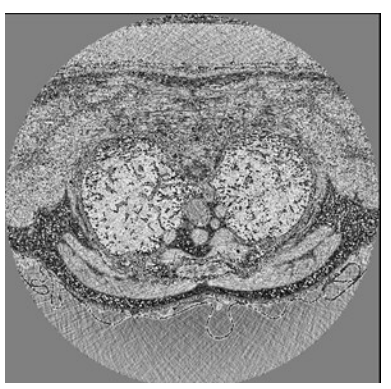

(A)

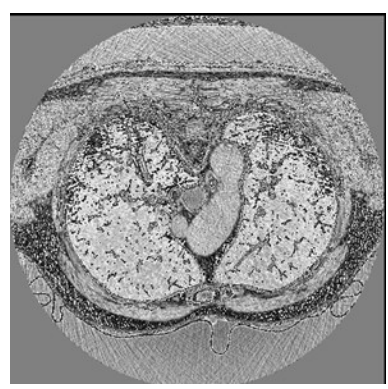

(B)
[3]. Based on this information a segmentation process with at least five clusters is needed for a normal data set.

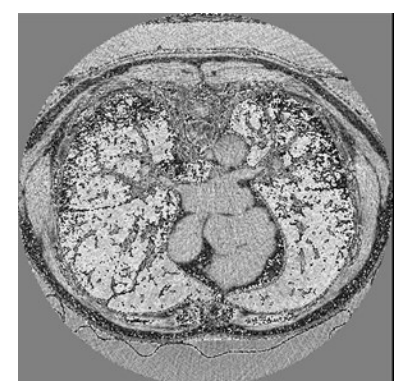

(C)

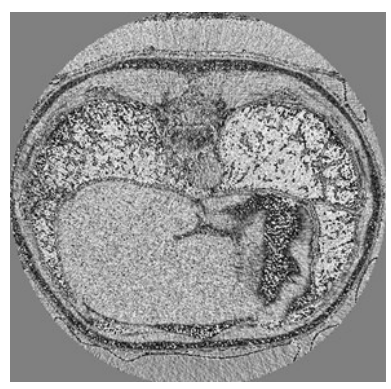

(D)

Figure 1. A, B, C and D show traverse Transverse Slices at levels 21, 40, 80 and 120, respectively, in the stack of the case under study.

\subsection{Lung Region Extraction}

In our previous work [4], we have developed an approach for the extraction of lung region from 3D CT chest image using bit plane slicing representation of each 2D CT image. The resulted binary slides are then analyzed to select best lung region's edge bit Plane image among them, bit-plane-2 was found to be the best representation for the used database.

Basic image processing techniques such as Erosion, median filter and dilation steps were used to remove inappropriate particulars that may augment additional complications to the lung region extraction process. We have improved this pre-extraction process in [5] as shown in Figure 2. The analysis of a larger database of CT chest images has shown that considering bit-plane-2 or bit-plane-1 in the pre-extraction process save better the fine lung borders in raw image, as shown in Figure 3. The result of the enhanced lung region extraction method as applied to the slices shown at Figure1 is given in Figure 4.

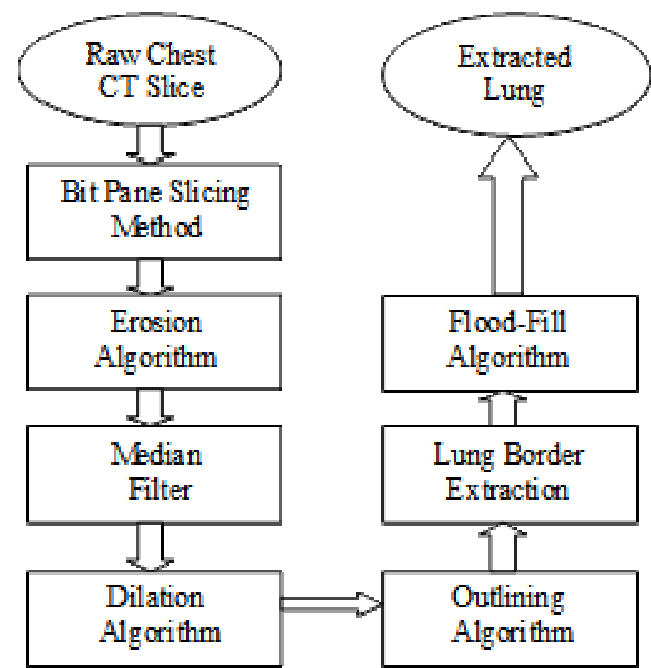

Figure 2. Bit Plane based extraction process for a $2 D$ CT slice using bitplane-2

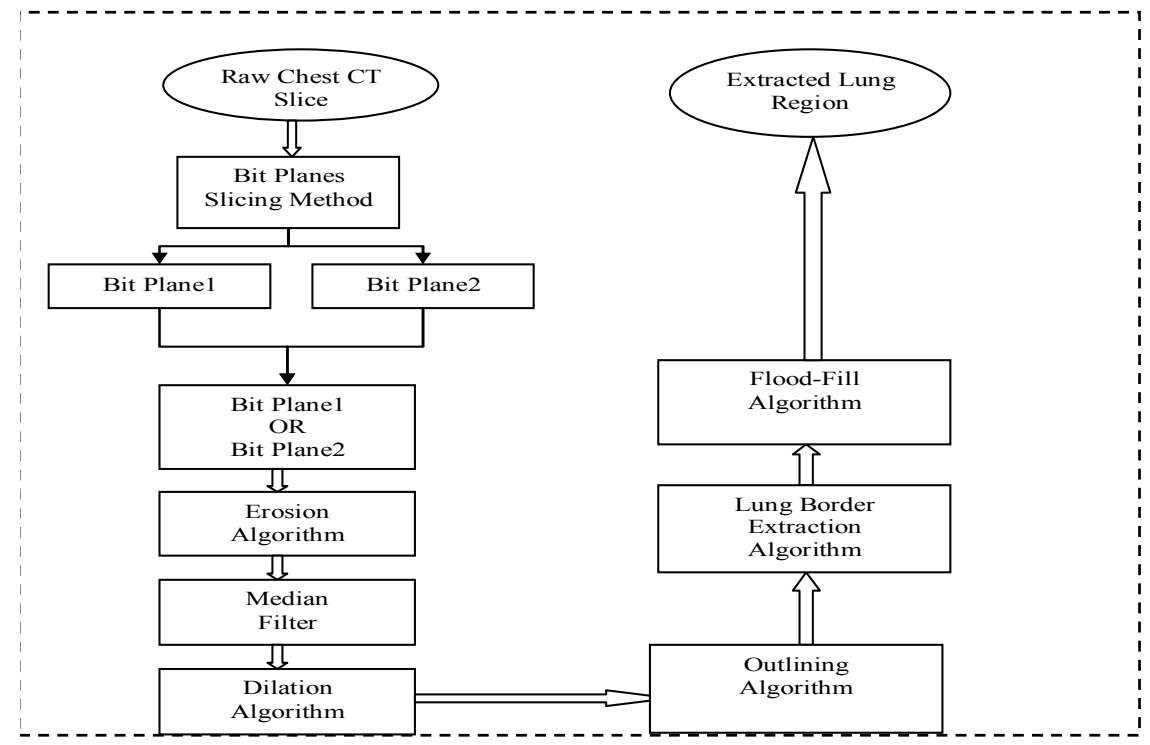

Figure 3. Modified Bit Planes based extraction process for a 2D CT slice using Bit Plane1 or Bit Plane2. 


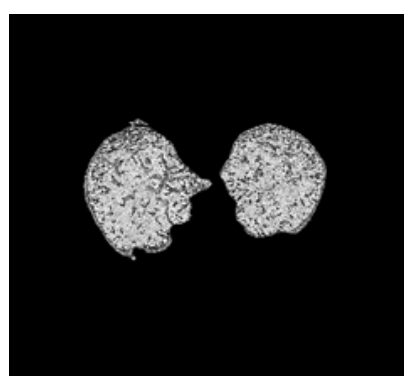

(A)

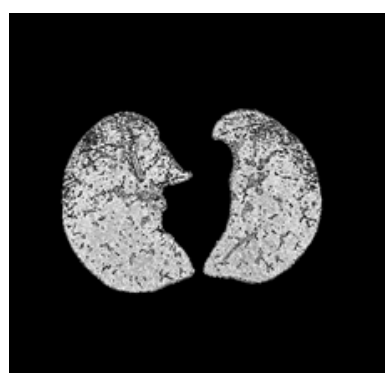

(B)

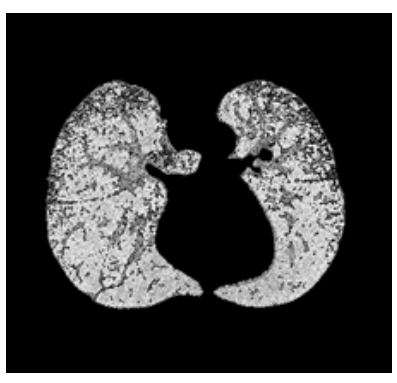

(C)

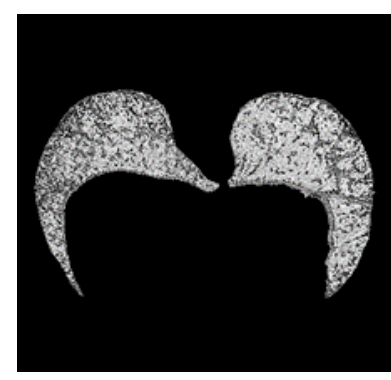

(D)

Figure 4. A, B, C and D show the extracted lung region using our modified bit-plane method applied to the transverse Slices at levels 21 , 40, 80 and 120, respectively, in the stack of the case under study shown in Figure 1.

When seen on transverse CT slices, the anterior and posterior junctions between the left and right lungs appear very thin and with low contrast. Gray-scale thresholding fails to separate the left and right lungs near these junctions [3]. However, using our technique described in [5] with more than $1000 \mathrm{CT}$ slices at different level in chest as shown in Figure 4 succeeded to separate sharply the regions of left and right lungs in most of the cases of our large CT database images without any pre-processing or modification to the raw data of the CT images.

\subsection{Lung Region Segmentation}

Image segmentation plays an important role in the screening of medical imaging. In that, improper segmentation of the Lung parenchyma will highly affect the accuracy of the Lung nodule detection or lung diseases classification algorithms. A recent review on adaptive segmentation of the pulmonary lobes and tumor identification from chest CT Scan Images is presented in [6], where the segmentation techniques are classified as semi-automatic algorithm and still need some user interaction, while others are fully automatic and the user has only a verification role.

Among the cited methods, we may brief here some of their main features or basic used techniques such as: A segmentation method suggested using artificial neural networks to classify each pixel in the CT slice into different anatomical structure supported by pre-processing and postprocessing techniques [7]. Another, have used anatomy guided 3D watershed transform for lung lobe segmentation, and a method of global thresholding for the segmentation purpose [8]. The results of these methods are promising and need enhancement in processing raw data of the CT images, and also improvement in the automation of the segmentation process as a subsystem of the CAD system.

In this section we present a modified Unsupervised Hopfield Neural Network Classifier (UHNNC) to segment 3D CT data set organized as a stack of 2-D transverse slices of $2 \mathrm{~mm}$ thin, where each pixel CT intensity value is stored as a short integer, collected from large databases of normal and cancerous lung tissues. The UHNNC architecture consists of a grid of $\mathrm{N} \times \mathrm{M}$ neurons with each column representing a class and each row representing a pixel, $\mathrm{N}$ represents the size of the given image and $\mathrm{M}$ represents the number of classes that is given as a priori information. The network is designed to classify the feature space without teacher based on the compactness of each class calculated using a distance measure. The segmentation problem is formulated [9] as a partition of $\mathrm{N}$ pixels of $\mathrm{P}$ features among $\mathrm{M}$ classes, such that the assignment of the pixels minimizes the cost-term of the energy (error) function as follows:

$$
E=\frac{1}{2} \sum_{k=1}^{N} \sum_{l=1}^{M} R_{k l}^{n} V_{k l}^{2}
$$

where $R_{\mathrm{kl}}$ is, a similarity distance measure between the $k^{\text {th }}$ pixel and the centroid of class $l$, defined as follows:

$$
R_{k l}=\left\|X_{k}-\bar{X}_{l}\right\|
$$

Where $\mathrm{X}_{\mathrm{k}}$ is, the $\mathrm{P}$-features vector, here $\mathrm{P}=1$ as the pixel's component is only the CT intensity value, of the $\mathrm{k}^{\text {th }}$ pixel, and $\bar{X}_{l}$ is, the centroid of class $l$, defined as follows:

$$
\bar{X}_{l}=\frac{\sum_{\mathrm{k}=1}^{\mathrm{N}} \mathrm{X}_{\mathrm{k}} \mathrm{V}_{\mathrm{kl}}}{\mathrm{n}_{\mathrm{l}}}
$$

where $n_{l}$ is the number of pixels in class $l$. In case of $\mathrm{n}=2$, the energy function of the UHNNC (1) could be considered as, the sum of squared errors that have been committed, during the assignment of the total pixels of the image, to the decided number of clusters during the classification or segmentation process. $V_{k l}$ is the output of the $k l^{t h}$ neuron. UHNNC uses the winner-takes-all learning as the inputoutput function for the $k^{\text {th }}$ row to assign a label $m$ to the $k^{t h}$ pixel and is given by:

$$
\left\{\begin{array}{rrr}
V_{k l}(t+1)=1, & \text { if } & U_{k l}=\operatorname{Max}\left\{U_{k l}(t), \forall l\right\} \\
V_{k l}(t+1)=0 ; & \text { otherwise }
\end{array}\right.
$$

The optimization of the energy function is achieved by solving a set of motion equations satisfying:

$$
\frac{\partial \mathrm{U}_{\mathrm{i}}}{\partial \mathrm{t}}=-\mu(\mathrm{t}) \frac{\partial \mathrm{E}}{\partial \mathrm{V}_{\mathrm{i}}}
$$

Where $U_{i}$ and $V_{i}$ are respectively the input and the output of the $i^{\text {th }}$ neuron, $\mu(t)$ is a scalar positive function of time used to increase the convergence speed of the UHNNC as:

$$
\mu(t)=t *\left(T_{s}-t\right)
$$

By applying the relation (5) to equation (1), we get a set of 
neural dynamics given by:

$$
\frac{d U_{k l}}{d t}=-\mu(t)\left(R_{k l}^{n} V_{k l}\right)
$$

The UHNNC segmentation algorithm can be summarized in the following steps:

1. Initialize the input of neurons to random values.

2. Apply the input-output relation given in (4) to obtain the new output value for each neuron, establishing the assignment of pixel to classes.

3. Compute the centroid for each class with respect to the equation (3)

4. Solve the set of differential equation in (5) to update the input of each neuron as:

$$
U_{k l}(t+1)=U_{k l}(t)+\frac{d U_{k l}}{d t}
$$

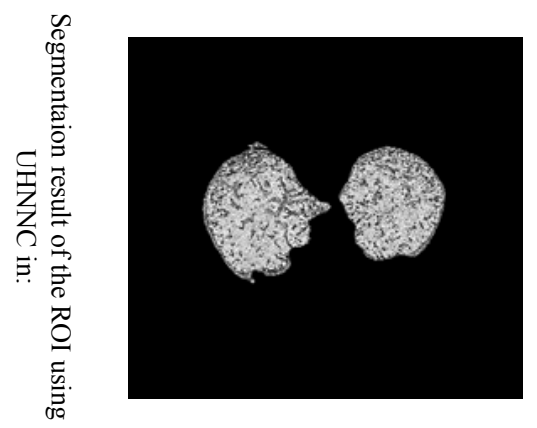

Slice 21 in the case

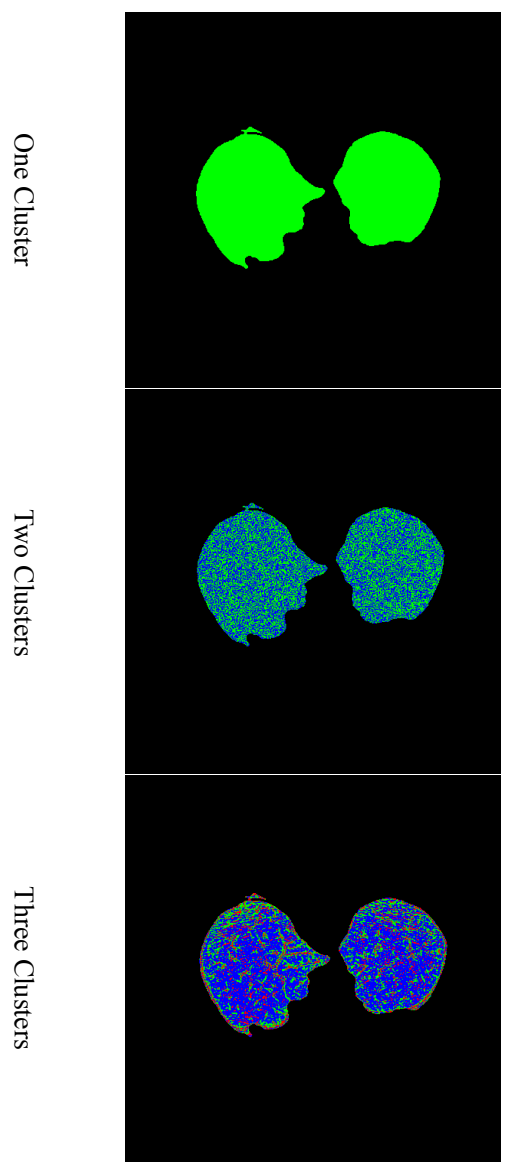

Figure 5. shows the segmentation results of the ROIs shown in Figure 4, using UHNNC in one, two and three clusters.

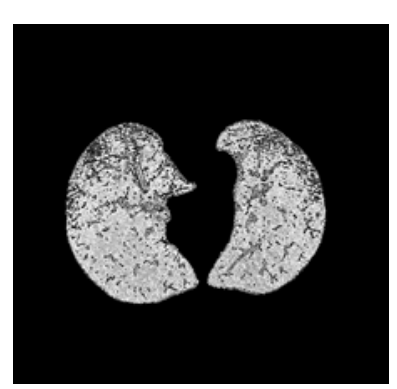

Slice 40 in the case
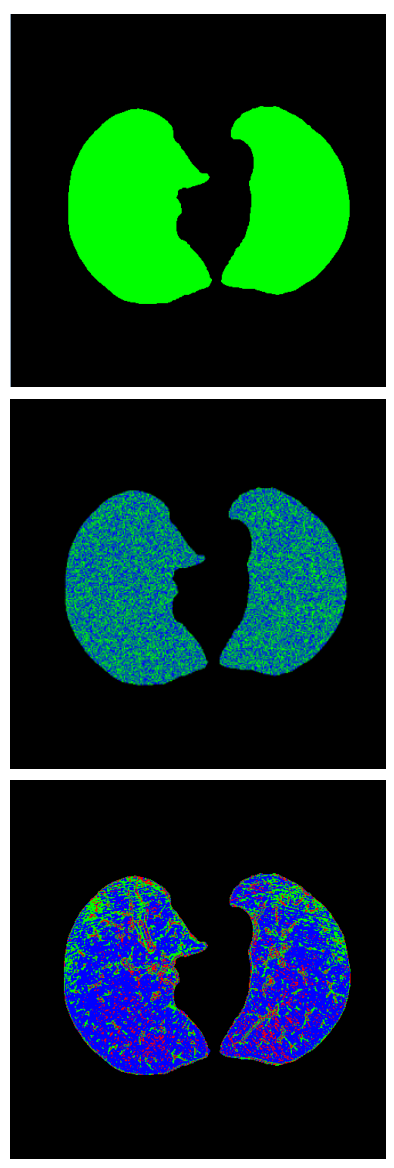

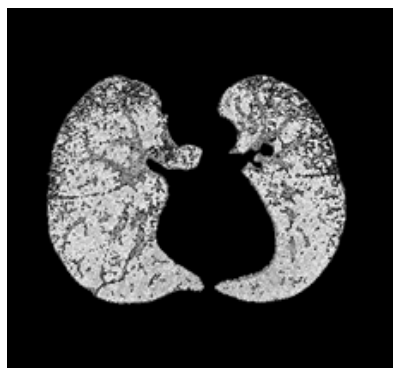

Slice 80 in the case
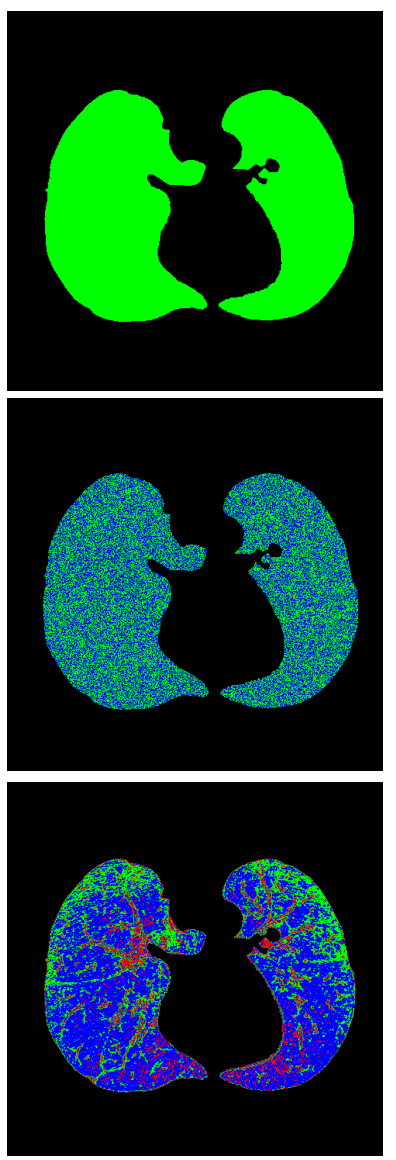

5. Repeat from step 2 until convergence then terminate.

We applied the above described UHNNC to several hundred of CT slices that have been chosen for this study. Our algorithm could segment $97 \%$ of the images successfully as it is shown in the next result section.

\subsection{Segmentation Results using UHNNC}

The segmentation process was initially developed and tested on a large dataset of subjects, with normal and abnormal lung tissues at different stages, each of $150 \mathrm{CT}$ scans giving very satisfactory results. Figure 5 shows the segmentation results of the ROIs shown in Figure 4, using the above described UHNNC with a parameter of the numbers of clusters between one and three. As can be seen, the segmentation results start to have a real matching with the raw CT data, when prepared with three clusters.

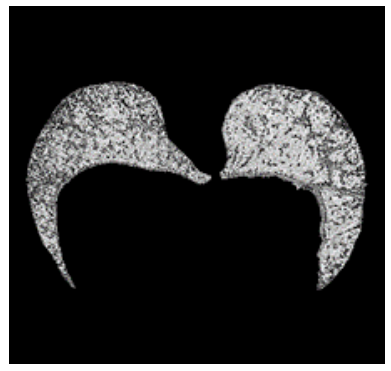

Slice 120 in the case
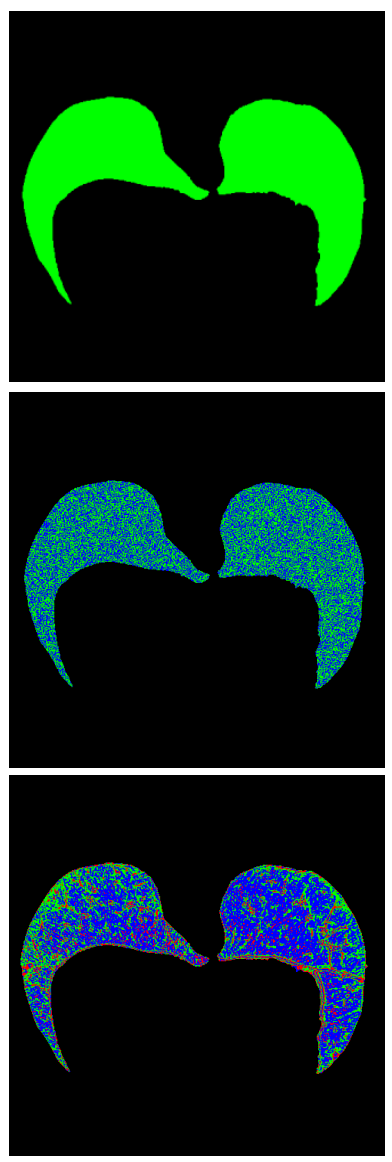
More details and reflections of the intensity variations in the ROIs could be detected in the segmentation results, if we increase the number of clusters as interactive parameter in the UHNNC. Figure 6 shows the segmentation results of the ROIs shown in Figure 4, using UHNNC in four, five and six clusters. Of course, we can increase the number of clusters as much as we want, and we will not have a problem with empty clusters. That is due to the intensity variations of the pixels among lung's tissues. Figure 7 shows the detailed information of the six clusters, of the slice 80 in the study case, obtained using the above described UHNNC.

\subsection{Discussion of the Segmentation Results using UHNNC}

We have developed segmentation method based on Unsupervised Hopfield Neural Network of Regions of Interests from chest CT images. The number of clusters is decided by the user based on medical information such as, number of tissues of the lung. The method produces as binary images as the number of clusters, where each pixel of the raw image is assigned to only and only one cluster, based on the pixel's intensity value and the mean value of the cluster, as shown in Figure7. The segmentation results show big matching between the raw and segmented images. However, more investigations have to be done to finalize the main clusters, to be used for analysis in the next stage of the CAD system.

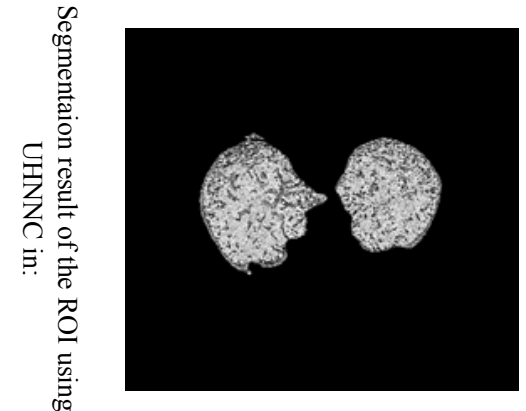

Slice 21 in the case

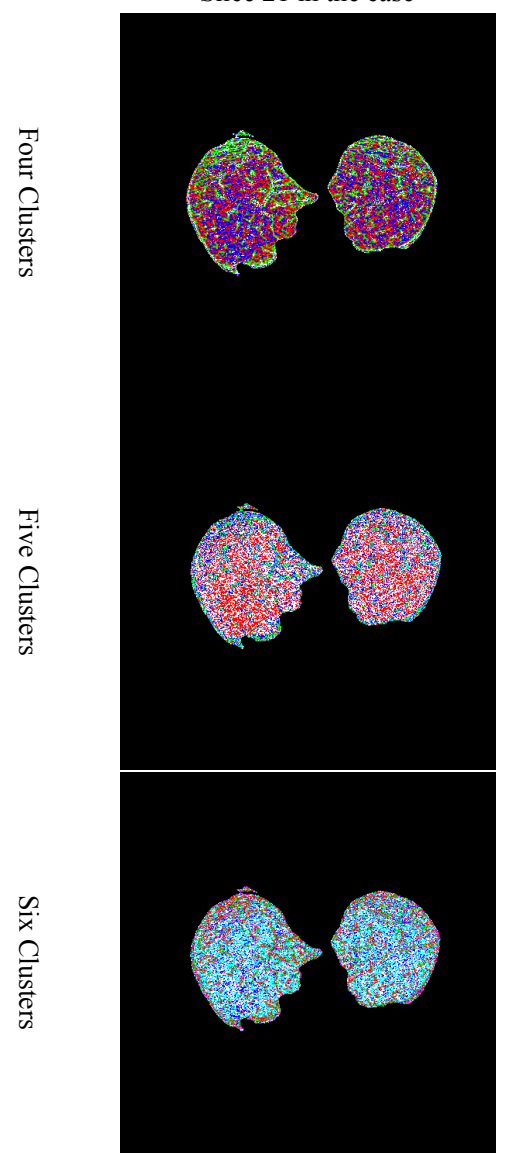

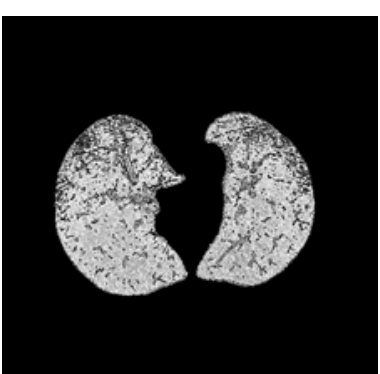

Slice 40 in the case
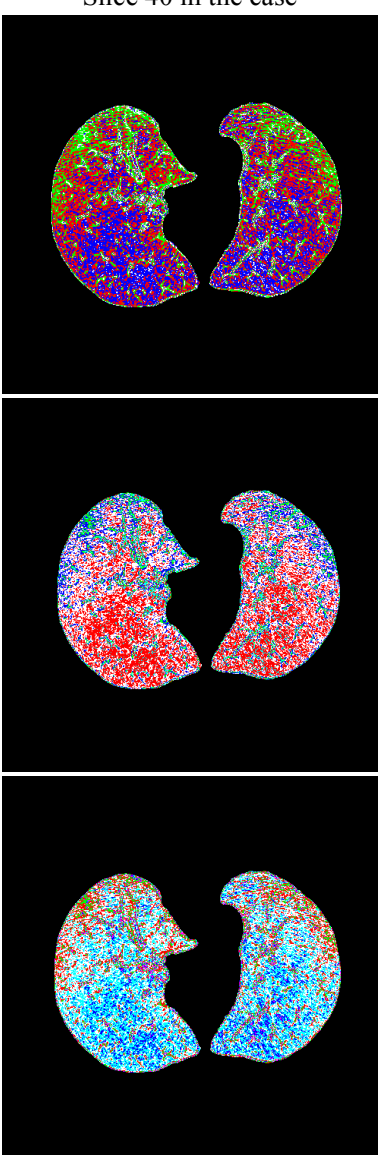

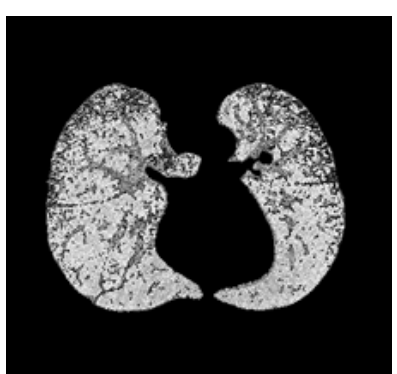

Slice 80 in the case
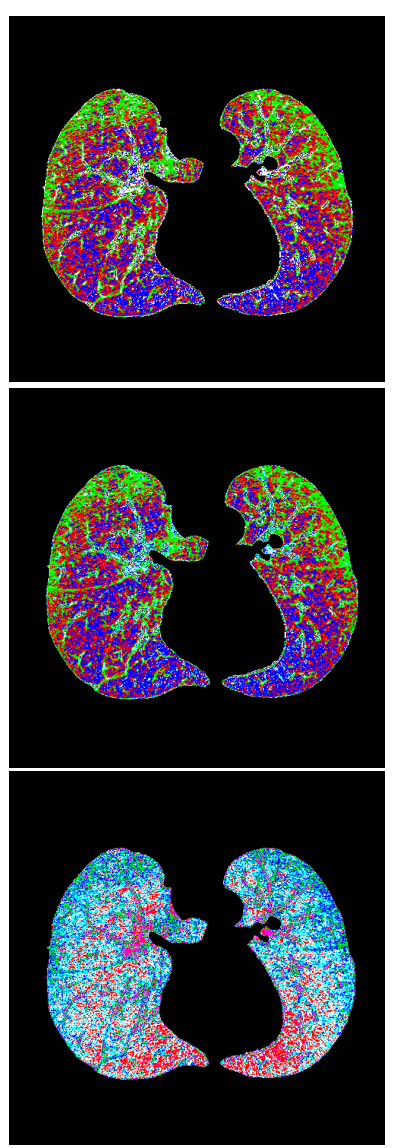

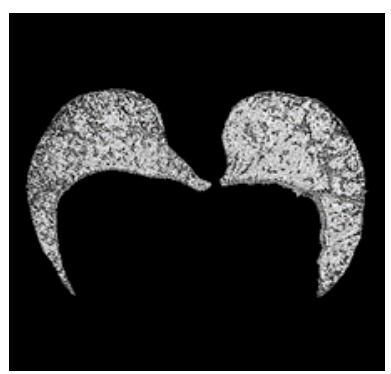

Slice 120 in the case
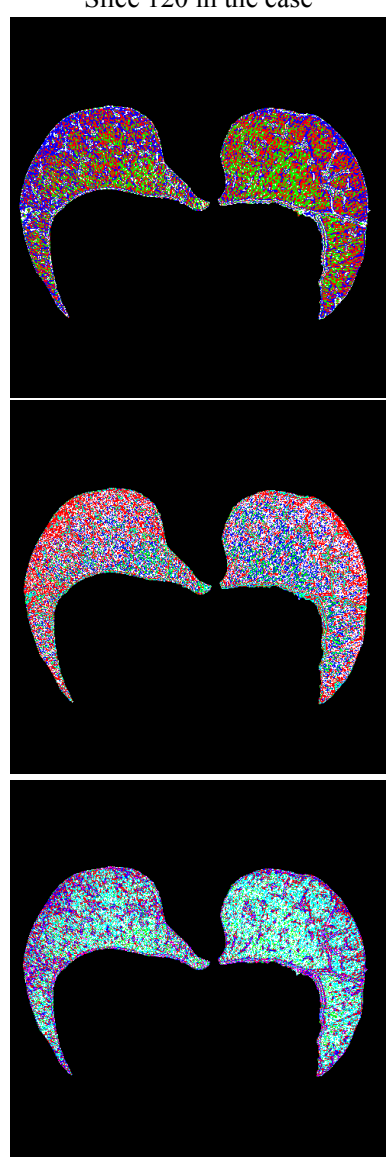

Figure 6. shows the segmentation results of the ROIs shown in Figure 4, using UHNNC in four, five and six clusters. 


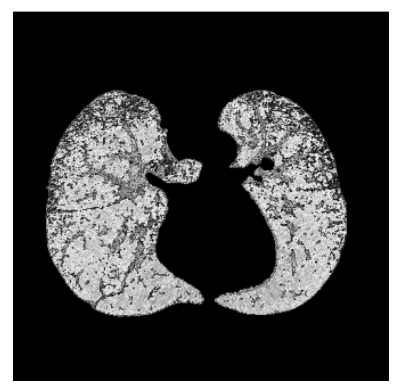

(a): Slice 80 in the case under study

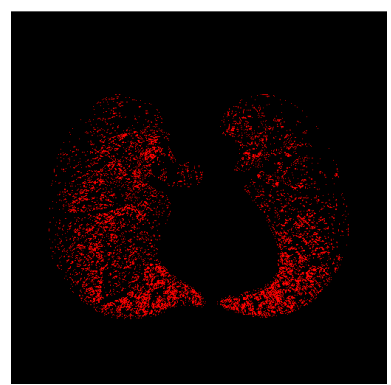

(e): Cluster No.3 in (b)

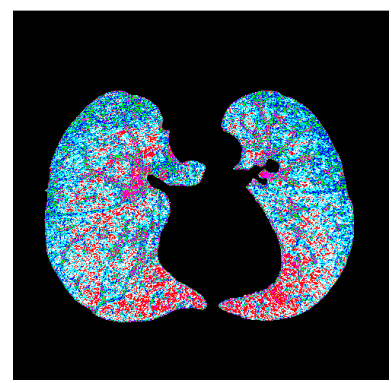

(b): Segmentation result with 6 clusters using UHNNC

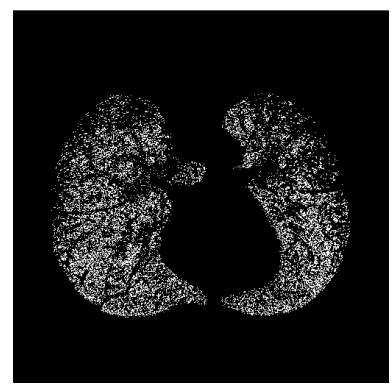

(f): Cluster No. 4 in (b)

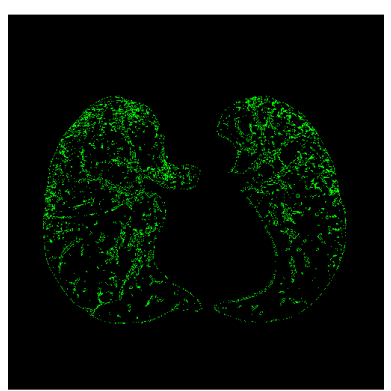

(c): Cluster No.1 in (b)

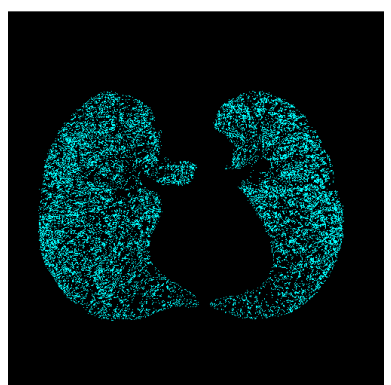

(g): Cluster No.5 in (b)

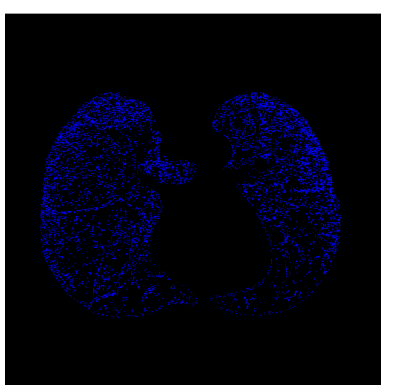

(d): Cluster No.2 in (b)

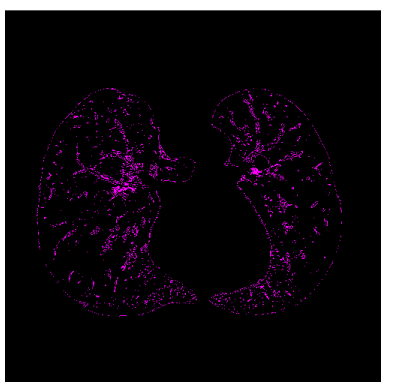

(h): Cluster No.6 in (b)

Figure 7. shows the segmentation result of the slice 80, in the study case, using UHNNC with the detailed information in its six clusters.

\section{Conclusion}

In this paper, we have presented an Unsupervised Hopfield Neural Network Classifier used for the segmentation of lung region in the Chest CT images. The segmentation results were satisfactory and appreciated by radiologists, as the obtained clusters show unique representations of the morphology of the different lung tissues. The intensity variation of the pixels in the raw CT slices makes decision of a final number of clusters unpredictable without the consideration of medical information about the possible number of different tissues in the ROIs. In our future work, we will focus on a post-segmentation process to refine the clusters, obtained by the UHNNC, in order to select the cancerous candidate regions with higher precision.

\section{Acknowledgment}

The authors would like to gratefully acknowledge the support for this research provided by the King Abdulaziz City for Science and Technology (KACST) under project number: "AL-11-0357" and the College of Computer and Information Sciences at King Saud University for hosting this project.

\section{References}

[1] The National Women's Health Information Center, U.S Department of Health and Human Services office on Women's Health, Lung Cancer, "http://www.4woman.gov/faq/lung.htm", 2003.
[2] Muhammad Usman1, Muhammad Shoaib2, and Mohamad Rahal1, "Multi-resolution Analysis Technique for Lung Cancer Detection in Computed Tomograpic Images", Progress In Electromagnetics Research Symposium (PIERS) Proceedings, Stockholm, Sweden, 1684-1688, Aug. , 2013.

[3] Shiying Hu, Eric A. Hoffman, "Automatic Lung Segmentation for Accurate Quantitation of Volumetric X-Ray CT Images", IEEE Transactions on Medical Imaging, Vol. 20, No. 6, June 2001.

[4] Rachid sammouda, Mohammed sammouda and Jamal Abu Hasan, "Automatic lung Region Extraction Algorithm from 3D CT-Images Based on Bit-Plane Slicing Technique", University of Sharjah Journal of Pure and Applied Sciences, vol.3, pp. 13-32, 2006.

[5] Rachid sammouda, Hassan Ben Mathkour and Ameur Tuoir, "Effect of Bit-Planes on the Extarction of Lung Region from 3D Chest CT Images", Journal of Advances in Computer Sciences and Engineering, vol.12, No. 2, pp. 119-128, 2014.

[6] Harsha Bodhey and Dr. G. S. Sable, "Review on: Adaptive Segmentation of the Pulmonary Lobes and Tumor Identification from Chest CT Scan Images", International Journal of Advanced Research in Computer and Communication Engineering, Vol. 2, Issue 10, pp. 4068-4071, October 2013.

[7] Prashant Naresh, Dr. Rajashree Shettar, "Early Detection of Lung Cancer Using Neural Network Techniques", Int. Journal of Engineering Research and Applications, ISSN : 2248-9622, Vol. 4, Issue 8( Version 4), August 2014, pp.78-83.

[8] Kalyani. G. Kohale \& Mahesh. S. Pawar, "Segmentation of Lungs Lobes and Distinction between Bening and Malignant Nodules by Use of Massive Training Artificial Neural Network for Surgical Preplanning", ITSI Transactions on Electrical and Electronics Engineering (ITSI-TEEE), ISSN (PRINT): 2320 - 8945, Volume -1, Issue -6, 2013. 
[9] Rachid Sammouda "Data Dependent Weight Initialization in the Hopfield Neural Network Classifier: Application to
Natural Colour Images", Journal of Computers and Applications, Vol. 32, No.2, 2010. 